\title{
A note from the Editorial Office
}

As we begin the year 2011, we first would like to thank all of our editorial board members, reviewers, authors, readers and collaborators for their great support, efforts and contributions to Chinese Science Bulletin (CSB) in promoting the journal's development over the past year, and wish everybody a happy New Year. This editorial will briefly introduce two important events that occurred in 2010, give a short report of the progress made in 2010, and describe some new developments for the coming year.

\section{Two important events in 2010}

The year 2010 marked the 60th anniversary of the publication of CSB. Founded in 1950, and co-sponsored by the Chinese Academy of Sciences (CAS) and the National Natural Science Foundation of China (NSFC), the journal has witnessed many important steps and achievements in science and technology in China. As a multidisciplinary journal with a 60-year history, CSB has published many important and well-known articles and has earned a high reputation among Chinese authors, reviewers and its broad readership. CSB has been one of the leading journals among the thousands of academic journals in China and has received many outstanding awards from the General Administration of Press and Publication of China, CAS and NSFC.

Another significant development for CSB was that the journal changed from semi-monthly (24 issues annually) to thricemonthly (36 issues annually) publication. This was historic in that the frequency of publication, having changed from monthly to semi-monthly, and then to thrice-monthly, may even be changed to weekly publication in the near future. Such changes are in good accordance with the development of the journal itself (such as its growing number of submissions and shortening publication cycle) in recent years as well as the need of scientists to publish their increasing numbers of high quality articles.

\section{Progress made in $\mathbf{2 0 1 0}$}

\subsection{Impact factor, total cites and the number of current articles}

According to the latest JCR report, CSB's impact factor reached 0.917 in 2009, an increase of $34 \%$ compared with 0.683 in the previous year. In the category of multidisciplinary sciences, CSB occupied 19th place and was in the second quartile. Total citations have reached 5116, ranking 10th of the total 50 journals. This success is the result of the efforts of all of the authors, reviewers, editors, and editorial board members. However, most encouragingly, as well as the increased IF and number of citations, CSB also has a relatively large author group, as evidenced by the number of current articles, which was 631 just behind the four most well-known journals: Proc Natl Acad Sci USA (3765), Ann NY Acad Sci (1101), Science (897) and Nature (866). We are very proud to be the fifth ranked journal in this category, and to be able to provide a larger platform for researchers to display their original findings, communicate their results and exchange ideas. Of course, we will continuously make every effort to raise the quality of published papers. We hope that CSB will become increasingly attractive to both young and established researchers around the world as a venue to publish their work.

\subsection{Downloads from SpringerLink}

Since the journal began its partnership with Springer in 2006 it has been moved to the SpringerLink online platform to promote its visibility worldwide. The increase in the number of times CSB articles were downloaded, which went from 6665 in 2006 to 188134 in 2009, was a tremendous jump. That number is estimated to continue to increase in 2010. Hopefully, in addition to increased visibility, this will also bring broader recognition to CSB.

\subsection{Professional English editing service}

English expression has long been difficult for most Chinese authors wishing to publish their articles in English. As a China-based journal, CSB has encountered difficulties in dealing with those papers with poor English, which has resulted in some negative impact on the readability of the journal. In 2010, with the aim of improving the language quality and the readability of the journal, CSB began collaboration with a professional English editing company. In this way, the journal has im- 
proved its standing, and we hope this strategy will continue to raise the profile of both the authors and the journal.

\section{Outlook for 2011}

\subsection{Open Access}

Open Access is a hot topic for today's international publishers. Science China Press, together with its partner Springer, will test the Open Access publication model on CSB from 2011. We expect that this decision will bring good news and a higher profile not only to CSB itself, but also to its authors and readers. Open Access means that anyone worldwide can access the full text of all articles published in CSB, free of charge, at csb.scichina.com and www.springerlink.com. Most importantly, instead of the "author pays" model (in which authors are charged the publication fees), Science China Press will cover all the costs of Open Access publication. It is expected that, although the journal is new to Open Access, it will bring notable and encouraging changes to CSB.

\subsection{Pub ASAP channel}

As proposed by Editor-in-Chief Prof. XIA JianBai, starting in this year, CSB will open a fast track to publication scheme with the formal launch of its Pub ASAP (Publication As Soon As Possible) channel, which guarantees manuscripts of higher quality a shorter turnaround time. Manuscripts that qualify for the Pub ASAP channel may be evaluated in two weeks, with a final decision and publication in one month online and three months in print. We will prioritize these excellent works, with the aim of attracting more prominent scientists to publish their articles in CSB. We hope the Pub ASAP channel will be favored by scientists and that it functions well in the future, under the generous support of the Editorial Board. For regular manuscripts, we will still make every effort to provide the best possible service and assistance to all of our authors.

\subsection{New website}

To make CSB more international and visible, CSB renewed its website with a new look befitting an international journal. The new website is user-friendly, functional and efficient. In particular, we have added lists of Accepted Articles and Online First Articles to the website so that articles can have immediate exposure to our audience. Authors, reviewers, board members and staff can easily and efficiently access the Online Manuscript System, and the readers can download full articles freely via the Internet. The new website is expected to better serve the journal and all of its users.

Finally, thanks also go to all the editors who made great efforts and contributions to the Special Topics published in CSB in 2010. They are Prof. JIANG GuiBin from the Research Center for Eco-Environmental Sciences, CAS, who edited the topic on "Environmental applications and effects of engineered nanomaterials"; Prof. GONG LiuZhu from the University of Science and Technology of China, who edited the topic on "Asymmetric organocatalysis"; Prof. GUO ZhengTang from Institute of Geology and Geophysics, CAS, who edited the topic on "Climate changes in the past century"[1], Prof. YAO TanDong from the Institute of Tibetan Plateau Research, CAS, who edited the topic on "Glacial retreat and its impact on lakes in Tibetan Plateau"[2]; Prof. WU WeiHua from China Agricultural University, who edited the topic on "Phytohormone"[3], Prof. LU ZuHong from Southeast University who edited the topic on "Bioinformatics"; Prof. XU HongXing from Institute of Physics, CAS, who edited the topic on "Plasmonics"[4]; Prof. WANG GuanWu from the University of Science and Technology of China, who edited the Special Issue "Dedicated to Professor LIU YouCheng on the occasion of his 90th birthday”[5] as well as Huazhong University's Special Topic [6].

With the New Year, we renew our resolve to continue to strive toward our goal of becoming a more international and influential multidisciplinary academic journal.

Wang S W. The global warming debate. Chinese Sci Bull, 2010, 55: 1961-1962

Yao T D. Glacial fluctuations and its impacts on lakes in the southern Tibetan Plateau. Chinese Sci Bull, 2010, 55: 2071

3 Li C Y, Li J Y. Toward understanding the molecular mechanisms governing plant hormone actions: A brief introduction to the Major Research Program "Molecular mechanisms of plant hormone actions" funded by the National Natural Science Foundation of China (NSFC). Chinese Sci Bull, 2010, 55: 2197

4 Xu H X. Frontiers of plasmonics. Chinese Sci Bull, 2010, 55: 2599

5 Wang G W. Editorial. Chinese Sci Bull, 2010, 55: 2759

6 Li P G. Preface. Chinese Sci Bull, 2010, 55: 3373

\section{AN Rui, Ph.D.}

Executive Director, Editorial Office of Chinese Science Bulletin

Open Access This article is distributed under the terms of the Creative Commons Attribution License which permits any use, distribution, and reproduction in any medium, provided the original author(s) and source are credited. 